Netro-Psycbopbarmaka, Bd. 1

Riederer P. / Laux G. / Pöldinger W. (Hrsg.)

(C) Springer-Verlag Wien 1992

\title{
11
}

\section{Allgemeine Grundlagen der Pharmakokinetik und Pharmakodynamik}

\author{
T. Buclin und P. Baumann
}

\subsection{Einführung}

\subsubsection{Definitionen und Zielsetzungen}

Dieses Kapitel über Pharmakokinetik sollte nur als Einführung in ein Gebiet angesehen werden, das in anderen, sich ausschliesslich mit diesem Bereich beschäftigenden Werken umfassender beschrieben wurde (TOzER 1981, Rowland und Tozer 1989, Gilman et al. 1990, KıoTz 1983). Es behandelt die quantitativen Beziehungen, die zwischen der Verabreichung eines Medikamentes und seiner klinischen Wirkung bestehen. Die hier dargestellten Beispiele dienen nur zur Illustration, da die Kinetik und Dynamik einzelner Pharmakagruppen in den jeweiligen Kapiteln eingehend beschrieben werden. Die zwei folgenden Begriffe verlangen eine genaue Definition:

Die Pharmakokinetik befaßt sich mit den Zusammenhängen zwischen der Medikamentendosis, seiner Verabreichungsart und den Konzentrationen der aktiven Komponente am Wirkungsort oder Rezeptor: Da diese in den meisten Fällen nicht meßbar sind, werden sie vereinfachend den Konzentrationen des freien, nicht an Eiweiße gebundenen Wirkstoffes im Plasma gleichgesetzt.

Die Pharmakodynamik beschäftigt sich mit der Beziehung zwischen der Konzentration des Medikamentes am Wirkungsort und seiner klinischen Wirkung, wobei für die erstere aus praktischen Gründen wiederum meist die Plasmakonzentration eingesetzt wird.

Die sich mit diesen Begriffen beschäftigende Wissenschaft bietet eine Sprache zur Beschreibung des Schicksals eines Medikamentes in Abhängigkeit von der Zeit und zum Verständnis der Wirkungsmechanismen bei. Diese erklärt die interindividuellen Unterschiede in der Reaktion auf eine Behandlung, und liefert dazu eine physiologische oder pathologische Begründung. Desgleichen stellt sie ein Werkzeug zur individuellen Dosisanpassung dar (drug monitoring, mit oder ohne Plasmabestimmung der Wirk- 
stoffe). Sie hilft, den Einflußs bestimmter Krankheiten auf das Schicksal des Medikamentes im Organismus und auf seine Wirkung, aber auch Interaktionen zwischen Medikamenten zu begreifen.

Im folgenden werden zahlreiche Formeln dargestellt, die im Wesentlichen der Nomenklatur vom American College of Cuinical PharMACOLOGY (1982) angepaßst wurden.

\subsubsection{Begrenzung auf die lineare Kinetik}

Es soll hier nur die lineare Pharmakokinetik, d. h. die der ersten Ordnung berücksichtigt werden, welcher die meisten Medikamente gehorchen, zumindest im klinisch gebräuchlichen Dosisbereich. Nach diesem Prinzip ist die Durchflußgeschwindigkeit eines Medikamentes von einem Kompartiment des Organismus zum andern proportional der Konzentration im Ausgangskompartiment (Abb. 11.1)

$\frac{\mathrm{dC}}{\mathrm{dt}}=-\mathrm{K} \cdot \mathrm{C}$

$\mathrm{C}=$ Konzentration des Medikamentes im Kompartiment, zu einem bestimmten Zeitpunkt

$\mathrm{K}=$ Zeitkonstante für dieses Kompartiment.

Manche Medikamente wie Phenytoin, Alkohol und zu einem gewissen Grade auch Lithium und Desipramin, gehorchen diesem Prinzip nicht.

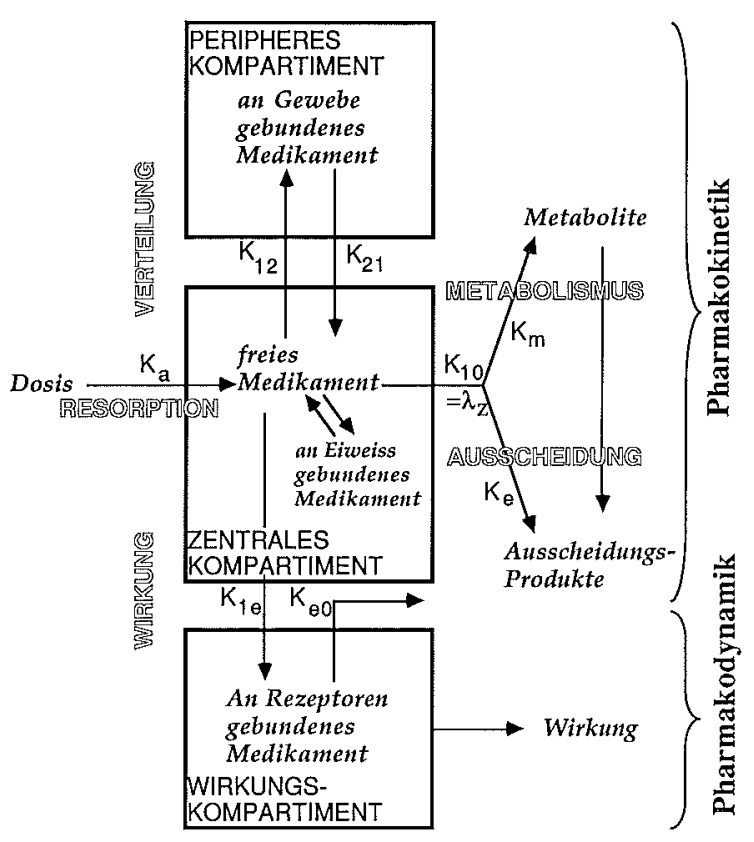

Abb. 11.1. Schema zum Schicksal der Medikamente im Organismus (Zweikompartimente-Modell mit "Wirkungskompartiment") 


\subsection{Kinetische Konzepte}

\subsubsection{Resorption}

Der Prozess der Resorption, auch Absorption oder Invasion genannt, entspricht der Wanderung des Medikamentes vom Verabreichungsort zum Blutkreislauf. Die erfaßten Parameter sind die Geschwindigkeit des Prozeßes sowie der Anteil des den Kreislauf erreichenden Medikamentes. Für ein nicht intravenös verabreichtes Medikament ist die Resorption stark von seiner galenischen Form abhängig, aber auch von seinem Verteilungskoeffizienten Wasser - Fett (Lipophilie), von seinem Ionisationsgrad, seinem Diffusionskoeffizienten (abhängig von der Molekülgröße) und von seiner Löslichkeit. Bei peroraler Verabreichung wird durch die verstoffwechselnde Rolle der Leber ein großer Anteil des durch die Pfortader transportierten Produktes eliminiert (First-pass-effect). Das Medikament kann aber auch durch die Galle wieder in den Darm ausgeschieden und sodann wieder über den Blutkreislauf in die Leber aufgenommen werden, was eine Verlängerung der Resorptionszeit zur Folge hat (enterohepatischer Kreislauf).

Die Resorption ist bei einer i.v.-Injektion in Form eines Bolus sofortig. Ihre Geschwindigkeit ist bei einer gleichmäßigen i.v.-Dauerinfusion konstant, d. h. die aufgenommene Menge ist proportional zur Perfusionsdauer (Kinetik nullter Ordnung). Bei einer Resorption auf nicht intravenösem Wege kann sie durch eine Kinetik erster oder nullter Ordnung oder durch eine nicht lineare Kinetik beschrieben werden. Eine Resorption kann manchmal erst nach Verzögerung auftreten, z. B. erst nach erfolgtem Übertritt vom Magen zum Darm. Dabei wird der vom verwendeten kinetischen Modell unabhängige Begriff Resorptionshalbwertszeit bevorzugt verwendet: er gibt das Zeitintervall an, während dem die Hälfte der resor- bierten Medikamentenmenge in den Blutkreislauf aufgenommen wurde.

Das Verhältnis aufgenommene Medikamentenmenge nach p.os versus i.v.-Gabe wird als Bioverfügbarkeit (f) bezeichnet. Dazu werden die Flächen unter den Kurven (AUC) verglichen, die einmal nach extravaskulärer und einmal nach intravenöser Applikation gemessen werden.

$\mathrm{f}=\frac{\mathrm{AUC}_{\text {extravaskulär }} \cdot \mathrm{D}_{\text {i.v. }}}{\mathrm{AUC}_{\text {i.v. }} \cdot \mathrm{D}_{\text {extravaskulär }}}$

$\mathrm{AUC}=$ Fläche unter der Kurve der beobachteten Konzentrationen

$\mathrm{D}=$ Applizierte Dosis

Zahlreiche psychotrope Pharmaka sind einem bedeutenden Firstpass-Effekt unterworfen. Somit ist ihre Bioverfügbarkeit zum Teil sehr gering: 30-50\% für Midazolam (GreenBLATT et al. 1983) und andere Benzodiazepine (Guentert 1984), ca 30-60\% für Neuroleptika wie Chlorpromazin, Flupentixol, Clopenthixol, Haloperidol (JøRGENSEN 1986) aber auch für trizyklische Antidepressiva (BREYERPfafF und GaERTNer 1987). Bei Unkenntnis der Biodisponibilität des Medikamentes ist es nicht möglich, die andern pharmakokinetischen Parameter einzig nach extravaskulärer Applikation zu gewinnen. Es können nur scheinbare Werte ermittelt werden. So hängt die scheinbare Clearance (CL') von der wahren Clearance (CL) ab, indem:

$\mathrm{f} \cdot \mathrm{CL}^{\prime}=\mathrm{CL}$

In der Psychopharmakologie wird bei Depotneuroleptika wie Haloperidoldecanoat die Resorption künstlich stark verlängert, sodaß sie zum geschwindigkeitsbestimmenden Schritt in der Kinetik des Medikamentes wird (Reyntjens et al. 1982). 


\subsubsection{Verteilung}

Gleich nach Einsetzen der Resorption etfährt das Medikament eine Verteilung im Organismus. Es erreicht zuerst den Blutkreislauf und die interstitielle Flüssigkeit der stark perfundierten Gewebe, deswegen auch als zentrales Kompartiment bezeichnet. Von da aus verteilt es sich in anderen Geweben, dem peripheren Kompartiment, je nach Affinität zu bestimmten Strukturen wie Fette oder Membranen des ZNS. Zahlreiche Arzneimittelmolekülle haben eine besondere Affinität zu verschiedenen Bestandteilen des Organismus, an die sie sich binden. Medikamente wie Benzodiazepine binden sich im Plasma bevorzugt an Albumin, basische wie Neuroleptika und Antidepressiva hauptsächlich an das saure $\alpha_{1}$-Glykoprotein und Lipoproteine (PIAFSKY 1983, Tillement und Lindenlaub 1986, Baumann et al. 1989). Da die meisten psychotropen Pharmaka zu 90-99\% an Plasmaeiweiße gebunden sind (Wandell und Wilcox-Thole 1983), ist von Bedeutung, daß nur der freie Anteil pharmakologisch wirksam und damit für die Diffusion in ein anderes Kompartiment, für den Abbau oder für die Eliminierung verfügbar ist. Die freie Fraktion $\left(f_{u}\right)$ wird definiert durch:

$f_{u}=\frac{C_{u}}{C}$

$\mathrm{C}_{\mathrm{u}}, \mathrm{C}=$ freie, resp. gesamte Konzentration des Medikamentes

Meistens mißt man die Gesamtkonzentration des Wirkstoffes im Plasma. Die pharmakokinetischen Parameter sind dementsprechend danach definiert. Falls die Bindung der Medikamente an Plasmaproteine bekannt ist, lassen sich die kinetischen Parameter in Funktion der freien Konzentrationen ausdrücken. So ist die freie Clearance $\mathrm{CL}_{\mathrm{u}}$ durch folgende Gleichung mit der Gesamtclearance CL verbunden:

$\mathrm{f} \cdot \mathrm{CL}_{\mathrm{u}}=\mathrm{CL}$
In den meisten Fällen genügt ein Zweikompartimente-Modell für die Beschreibung der Verteilung eines Medikamentes im Organismus. Die Kurve der Plasmakonzentrationen nach einer einmaligen Dosis zeigt zunächst einen raschen Abfall, entsprechend der Verteilungs- oder $\alpha$-Phase in das periphere Kompartiment. Sind einmal die beiden Kompartimente im Gleichgewicht, verlangsamt sich der Konzentrationsabfall, der Eliminations- oder $\beta$-Phase entsprechend.

Jedes Kompartiment ist vollständig definiert durch sein scheinbares Verteilungsvolumen und die Zeitkonstante, die es mit dem benachbarten Kompartiment verbindet.

Das scheinbare Verteilungsvolumen $V$ ist eine Zahl, ohne anatomische Entsprechung:

$\mathrm{V}=\frac{\mathrm{A}}{\mathrm{C}}$

$A=$ im Kompartiment vorhandene Arzneimittelmenge

$\mathrm{C}=\mathrm{im}$ Kompartiment gemessene Arzneimittelkonzentration

Bei einem Zweikompartimente-Modell (bei schneller i. v. Applikation) erhält man das Verteilungsvolumen des zentralen Kompartimentes $V_{c}$ durch Einbeziehung der extrapolierten, fiktiven Konzentration zum Zeitpunkt $O(C(0))$, also zu einem Zeitpunkt, bevor die Diffusion in das periphere Kompartiment begonnen hat:

$\mathrm{V}_{\mathrm{C}}=\frac{\mathrm{D}}{\mathrm{C}(0)} \quad \mathrm{D}=$ Dosis

Nach der Gleichgewichtseinstellung zwischen den beiden Kompartimenten kann man das Verteilungsvolumen während der Endphase $\left(V_{Z}\right)$ definieren:

$\mathrm{V}_{\mathrm{z}}=\frac{\mathrm{D}}{\lambda_{\mathrm{z}} \cdot \mathrm{AUC}}$

$\lambda_{z}=$ Zeitkonstante im Zusammenhang mit der Elimination des Medikamentes (s. u.)

$\mathrm{AUC}=$ Fläche unter der Kurve 
Bei kontinuierlicher oder wiederholter Applikation definiert man noch das Verteilungsvolumen im Fließgleichgewicht (steady state) $\left(V_{s s}\right)$, dessen Wert meist kleiner ist als $V_{z}$ (Tozer 1981). Bei einer sehr schnellen Verteilungsphase, oder zumindest bei einer, die schneller ist als die Resorptionsphase, die zum geschwindigkeitsbestimmenden Schritt wird, reicht in manchen Fällen ein Einkompartiment- Modell:

In einem Einkompartimentmodell sollten die genannten Volumen einander entsprechen: das Verteilungsvolumen $V_{d}$ wird nach der gleichen Formel wie $V_{c}$ berechnet, ausgedrückt in Litern pro kg Körpergewicht. Die Werte müssen immer höher als das reale Plasmavolumen sein, übersteigen aber oft in bedeutendem Maße das Volumen des gesamten Organismus. Sie hängen von der Affinität des Medikamentes für die Plasmaund Gewebeeiweiße ab: ist erstere hoch, ist das Verteilungsvolumen gering, aber je höher die Gewebsbindung desto grösser das Verteilungsvolumen. Das von Lithium und Benzodiazepinen ist verhältnismäßig geting, resp. 0,5-1,21/kg (AMDISEN 1983) und 0,3-1,7 1/kg Körpergewicht (GUENTERT 1984, GREENBLATT et al. 1983, CrisMon 1983), hingegen liegt das von Antidepressiva (DoBbs 1983, Breyer-Pfaff und GaErTner 1987) und Neuroleptika um 6-90 1/kg (JøRGENSEN 1986).

\subsubsection{Elimination}

Die Elimination des Medikamentes erfolgt durch Metabolisierung und Ausscheidung durch die Niere, im allgemeinen über eine Kinetik ersten Grades. Während der Eliminationsphase ist deshalb die pro Zeiteinheit ausgeschiedene Menge proportional zur Plasmakonzentration. Der Proportionalitätsfaktor ist durch die Gesamtclearance (CL) des Medikamentes definiert und mit folgender Gleichung mit der Zeitkonstante der Eliminationsphase $\left(n_{z}\right)$ direkt verbunden, welche das Verhältnis zwischen der Verän- derung der Konzentration pro Zeiteinheit und der Konzentration selbst darstellt:

$\mathrm{CL}=\lambda_{\mathrm{z}} \cdot \mathrm{V}_{\mathrm{z}}$

$\mathrm{V}_{\mathrm{z}}=$ Verteilungsvolumen der Endphase

CL kann auch als das Plasmavolumen interpretiert werden, welches pro Zeiteinheit vollständig vom Medikament befreit wird. Nach einer einmaligen i.v.-Applikation berechnet sich die Clearance nach:

$\mathrm{CL}=\frac{\mathrm{D}}{\mathrm{AUC}}$

$\mathrm{D}=$ injizierte Dosis

$\mathrm{AUC}=\mathrm{Fläche} \mathrm{unter} \mathrm{der} \mathrm{Kurve}$

Ein wichtiger Parameter ist auch die Eliminationshalbwertszeit $\left(t_{1 / 2}\right)$. Sie stellt die Zeit dar, während der die Plasmakonzentration um die Hälfte abnimmt. Im Falle einer wiederholten Applikation des Medikamentes mit einer Einkompartimentskinetik wird dieselbe $t_{1 / 2}$ verwendet, um die Geschwindigkeit der Einstellung des Fließgleichgewichtes (steady-state) anzugeben. Die Halbwertszeit $t_{1} / 2$ ist deshalb durch das Verteilungsvolumen in der Endphase und die Gesamtclearance des Arzneimittels definiert:

$\mathrm{t}_{1 / 2}=\frac{\ln 2}{\lambda_{z}}=\frac{0,693}{\lambda_{z}}=\frac{0,693 \cdot V_{z}}{C L}$

Die psychotropen Pharmalka unterscheiden sich in ihren terminalen (oder $\beta$-) Halbwertszeiten beträchtlich. Sie beträgt ca. 20 Stunden für Lithium (AMDisen 1983), für Antidepressiva (Breyer-PhafF und GaERTNer 1987) und Neuroleptika (JørGENSEN 1986) in der Regel 10-50 Stunden, wobei aber noch Extremwerte auftreten wie 2-5 Stunden für Medikamente wie Viloxazin und Lofepramin, ein "Prodrug" von Desipramin. Bei Benzodiazepinen wurde die Entwicklung bewußt dahin gesteuert, über Produkte mit sehr kurzen bis zu sehr langen Halbwertszeiten zu verfügen, von Triazolam und Midazolam mit Werten um 2-3 Stunden 
(GreEnBlatT et al. 1983) bis zu Diazepam und Desmethyldiazepam mit $t_{1 / 2}$ um 20-150 Stunden (Crismon 1983).

\section{Renale Ausscheidung}

Die Gesamtclearance setzt sich aus der renalen Clearance $\left(\mathrm{CL}_{R}\right)$ und der metabolischen, meist hepatischen Clearance $\left(\mathrm{CL}_{H}\right)$ des $\mathrm{Me}$ dikamentes zusammen:

$\mathrm{CL}=\mathrm{CL}_{\mathrm{R}}+\mathrm{CL}_{\mathrm{H}}$

Die renale Clearance, $C_{R}$, ist das Produkt aus der Zeitkonstante der renalen Ausscheidung, $K_{e}$, und dem Verteilungsvolumen in der Endphase, $V_{z}$. Sie wird ermittelt, indem man die ausgeschiedene Menge des unveränderten Medikamentes $\left(\mathrm{A}_{\mathrm{e}}\right)$ nach einer einmaligen Verabreichung berechnet:

$\mathrm{CL}_{R}=\frac{\mathrm{A}}{\mathrm{AUC}}$

Man kann sie auch mit dem Verhältnis der renalen Ausscheidungsgeschwindigkeitund der Konzentration in der Nierenarterie $\left(C_{A}\right)$ gleichsetzen:

$C_{R}=\frac{Q_{R} \cdot\left(C_{A}-C_{V}\right)}{C_{A}}$

$Q_{R}=$ plasmatische Durchflußgeschwindigkeit in der Niere

$\mathrm{C}_{\mathrm{V}}=$ Konzentration in der Nierenvene

Der Mechanismus der Nierenausscheidung ist die Resultante der passiven Filtrierung des Medikamentes durch die glomeruläre Membran, seiner passiven oder aktiven Wiederaufnahme durch die Tubuluswände der Niere, und seiner Sekretion, falls existierend, durch die renalen Tubuli. Bei Abwesenheit von sowohl Selsretion wie Reabsorption ist die renale Clearance $\left(C_{R}\right)$ das Produkt der freien Plasmakonzentration $\left(f_{u}\right)$ mit der glomurälen Filtrierung. Sie hängt somit von der Bindung des Arzneimittels an Bluteiweiße ab. Bei sauren oder basischen Medikamenten hängt die passive Wieder- aufnahme vom Ionisationsgrad des Moleküls ab, welcher selbst wiederum vom $\mathrm{pH}$ des Urins und von der Urindichte abhängt. Natürlich spielt gerade beim Lithium wegen der fehlenden Biotransformation die renale Ausscheidung eine bedeutende Rolle. Für Lithium beträgt die renale Clearance 10-40 $\mathrm{ml} / \mathrm{min}$ (AMDISEN 1983). Das Auftreten von aktiven Sekretions- und Reabsorptionsmechanismen erklärt die sättigbare Kinetik dieses Ions.

\section{Biotransformation}

Die Biotransformation, d. h. der Metabolismus von Medikamenten findet bevorzugt in der Leber und in geringerem Maße in der Niere, im Darm, in den Lungen, in der Plazenta oder im Blut statt. Die hepatische Clearance wird aus der Gesamtclearance und aus der renalen Clearance berechnet (s. oben). Sie ist dem Verhältnis zwischen der hepatischen Ausscheidung und der Konzentration im hepatischen Pfortadersystem, $\mathrm{C}_{\mathrm{A}}$, gleich zu setzen;

$\mathrm{CL}_{\mathrm{H}}=\frac{\mathrm{Q}_{\mathrm{H}} \cdot\left(\mathrm{C}_{\mathrm{A}}-\mathrm{C}_{\mathrm{V}}\right)}{\mathrm{C}_{\mathrm{A}}}$

Das Verhältnis $\left(\mathrm{C}_{A}-\mathrm{C}_{V}\right) / \mathrm{C}_{A}$ wird als hepatischer Extraktionskoeffizient $\left(\mathrm{E}_{\mathrm{H}}\right)$ bezeichnet. Er ist verhältnismäßig spezifisch für ein bestimmtes Medikament und mißt die metabolische Aktivität des Lebergewebes gegenüber diesem Medikament. Für solche mit einem hohen um 1 liegenden Koeffizienten zeigt die oben dargestellte Formel, daß die hepatische Clearance hauptsächlich von der Leberdurchblutung abhängt $\left(\mathrm{QH}_{\mathrm{H}}\right)$, welcher zum geschwindigkeitsbestimmenden Schritt der Ausscheidung wird. Da das gesamte Blut, das den Darm durchströmt, über die Pfortader durch die Leber fließt, muß man für die peroral verabreichten Medikamente mit einem bedeutenden "First-pass-effect" rechnen, so dass die maximale Biodisponibilität beträgt:

$\mathrm{f} \leq 1-\mathrm{E}_{\mathrm{H}}$ 
Für die Medikamente mit niedrigem, nahe bei 0 liegenden $\mathrm{E}_{\mathrm{H}}$ hängt $\mathrm{CL}_{\mathrm{H}}$ hauptsächlich von der verstoffwechselnden Aktivität der Leber und dem Bindungsgrad an Plasmaproteine ab. Diese beiden Faktoren sind selbst häufig verschiedenen pharmakokinetischen (Induktion, enzymatische Hemmung, Verdrängung von der Eiweissbindung) oder genetischen (Existenz von Untergruppen mit genetisch bedingter Verminderung des Metabolismus) Einflüssen ausgesetzt (s. Baumann, dieser Band). Die metabolische Aktivität der Leber selbst hängt im Sinne einer sättigbaren Kinetik nach Michaelis-Menten von der Konzentration des Medi- kamentes ab. Die meisten psychotropen Pharmaka werden in der Leber metabolisiert, in einer ersten Phase zunächst durch Oxidation mittels Cytochrom P-450, oder auch zum Beispiel durch Acetylierung wie im Falle von Nitrazepam. Bei Vorhandensein einer Hydroxygruppe oder nach erfolgter Oxidation zu einer Hydroxyverbindung findet eine Konjugierungsreaktion zur Bildung von Glucuroniden statt (Burchelr, und Covghtrie 1989). Natürlich sind zahlreiche Metabolite selbst auch aktiv. Auf die speziellen kinetischen Probleme soll hier aber nicht eingegangen werden.

\subsection{Pharmakokinetische Modelle}

Die Verabreichung eines Medikamentes nach einem bestimmten Schema führt zu einer Plasmakonzentration, deren Entwicklung zeitabhängig ist. Sie wird gewöhnlich graphisch logarithmisch (Abb. 11.1) und nach dem gewählten kinetischen Modell als mathematische Funktion, z. T. mittels Computerprogrammen dargestellt. Daraus werden die oben beschriebenen pharmkokinetischen Parameter berechnet. Thre Kenntnis erlaubt die Konzentrationskurven für andere Applikationsschemen zu entwickeln und für den individuellen Patienten die optimalen Dosen zu berechnen.

\subsubsection{Modell im Falle einer einmaligen i.v.-Applikation (Bolus) (Abb. 11.2)}

Im Falle eines Modelles mit zwei Kompartimenten ist die zur Beschreibung der Konzentrationsverläufe verwendete Formel:

$C=C_{1} \cdot e^{-\lambda_{1} t}+C_{2} \cdot e^{-\lambda_{2} t}$
Zum Zeitpunkt null, vor dem Beginn der Verteilungsphase liegt die Anfangskonzentration $(\mathrm{C}(\mathrm{O}))$ vor:

$\mathrm{C}_{(0)}=\mathrm{C}_{1}+\mathrm{C}_{\mathrm{z}}$

Die Integration der Kurve über den gesamten zeitlichen Raum gibt die Fläche unter der Kurve (AUC):

$\mathrm{AUC}=\int_{0}^{\infty} \mathrm{Cdt}=\frac{\mathrm{C}_{1}}{\lambda_{1}}+\frac{\mathrm{C}_{\mathrm{z}}}{\lambda_{\mathrm{z}}}$

Mittels dieser vier Begriffe $C_{1}, C_{z}, \lambda_{1}, \lambda_{z}$ können alle klassischen kinetischen Parameter nach den vorhergehenden Formeln abgeleitet werden.

\subsubsection{Fall einer oralen Einzeldosis (Abb, 11.2)}

Falls die Absorption einer Kinetik erster Ordnung unterliegt, so gilt nach dem Modell die Funktion:

$C=C_{1} \cdot e^{-\lambda_{1} t}+C_{z} \cdot e^{-\lambda_{z} t}-\left(C_{1}+C_{z}\right) \cdot e^{-K_{a} t}$ 
Die Schätzung der fünf Parameter dieses Modelles erlaubt nur die Darstellung scheinbarer Werte der kinetischen Parameter für ein gegebenes Medikament: für die tatsächlichen Werte sind die nach i.v. und per os erhaltenen AUC's notwendig, um ein korrektes Bild der Biodisponibilität zu gewinnen.

\subsubsection{Fall einer Dauerinfusion}

(Abb. 11.2)

Bei der Annahme, die Infusion beginnt zum Zeitpunlst null, erklärt folgende Formel den Verlauf der Konzentrationen:

$\mathrm{C}=\frac{\mathrm{C}}{\lambda_{1} \mathrm{~T}}\left(1-\mathrm{e}^{-\lambda_{1} \mathrm{t}}\right)$

$\mathrm{T}=$ Dauer der Infusion.

Nach einer langdauernden Infusion erreicht C ein steady- state, $\mathrm{C}_{\mathrm{ss}}$ :

$\mathrm{C}_{\mathrm{ss}}=\frac{\mathrm{C}_{1}}{\lambda_{1} \mathrm{~T}}=\frac{\mathrm{R}_{0}}{\mathrm{CL}}$
$\mathrm{R}_{0}=$ Menge des pro Zeiteinheit infundierten Medikamentes

Die Messung von $\mathrm{C}_{\mathrm{ss}}$ erlaubt deshalb die Ableitung von $\mathrm{C}_{\mathrm{L}}$. Das Verteilungsvolumen im steady-state, $V_{s s}$, kann ebenfalls berechnet werden. Es ist kleiner als das Verteilungsvolumen in der Endphase. Man stellt fest, daß $V_{s s}$ nicht in die Berechnung von $C_{s s}$ eingeht.

\subsubsection{Fall einer wiederbolten oralen Applikation (Abb. 11.2)}

Die in dieser Situation erhaltene Kurve ist kompliziert (Abb, 11.2). Man nähert sich progressiv einem Fließgleichgewicht um eine mittlere (average) Konzentration, $\mathrm{C}_{s s}$ av:

$\mathrm{C}_{\mathrm{ss} \text { av }}=\frac{\mathrm{f} \cdot \mathrm{D}}{\mathrm{CL} \cdot \tau}=\frac{\mathrm{AUC}_{\mathrm{SS} \tau}}{\tau}$

$\mathrm{f}=$ Biodisponibilität $\mathrm{D}=$ Einzeldosis $\tau=$ Zeit intervall zwischen zwei Dosen $\mathrm{AUC}_{S S \tau}=$ Fläche unter der Kurve während eines einzi-
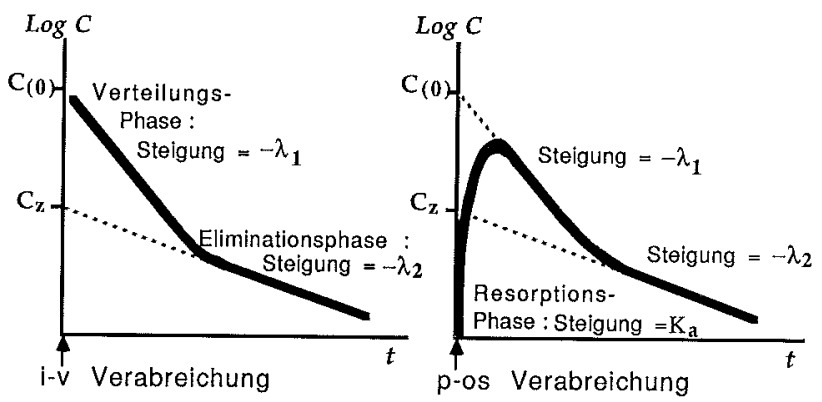

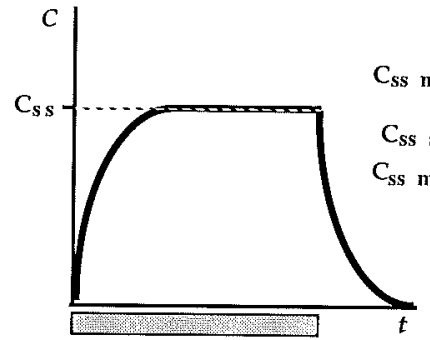

Konstante i-v-Infusion

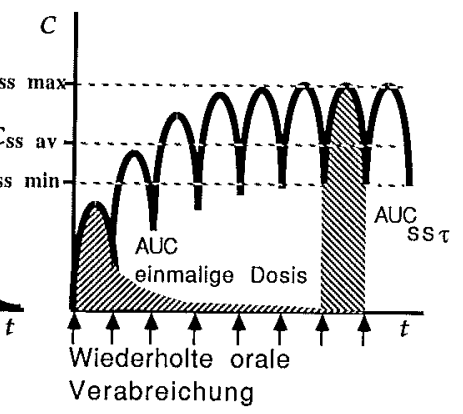

Abb. 11.2. Konzentrationskurven in vier klassischen Verabreichungssituationen 
gen Dosisintervalls = Fläche unter der Kurve nach einer Einzeldosis.

Im steady-state oszillieren die Konzentrationen zwischen einem Minimum und einem
Maximum, im Falle eines Einkompartimentmodells:

$$
\mathrm{C}_{S S \max }=\frac{\mathrm{f} \cdot \mathrm{D}}{\mathrm{Vd}_{\mathrm{d}} \cdot\left(1-\mathrm{e}^{-\lambda_{\mathrm{z}} \tau}\right)}
$$

\subsection{Dynamische Konzepte}

\subsubsection{Konzentrations - Wirkungsbeziebung}

Die Plasmakonzentration eines Medikamentes ist ein gewisses Maß für seine Aktivität nach Verabreichung einer bestimmten Dosis. In manchen Fällen stellen diese Konzentrationen ein getreues Abbild der Konzentrationen am Wirkungsort (Rezeptor) dar; welche in einer quantitativen Beziehung zur Intensität der pharmakologischen Wirkung stehen. In Situationen, in denen die Wirkung sich auf eine binäre Response-Möglichkeit "Erfolg - Nichterfolg" beschränkt, steht die Erfolgswahrscheinlichkeit in einer Gruppe von behandelten Patienten in direkter Beziehung zur Konzentration.

Üblicherweise stellt man diese Konzentrations-Wirkungs-Beziehung durch eine logarithmische Skala wie in Abb. 11.3 dar. Im mittleren Teil der Kurve ist sie oft linear, etwa zwischen $20-80 \%$ der maximalen Wirkung, obwohl auf den sigmoidalen Charakter der Gesamtkurve hingewiesen werden muß. Tatsächlich gibt es immer eine maximale Wirkung $\left(\mathrm{E}_{\max }\right)$, oberhalb der selbst eine höhere Konzentration keine weitere Steigerung des Effektes mehr erzielt und die die Eigenwirkung oder die Wirksamkeit des Arzneimittels definiert. Die Plasmakonzentration, bei der $50 \%$ der maximalen Wirkung erzielt wird, heisst wirksame Konzentration $50\left(\mathrm{EC}_{50}\right)$ und gibt das Maß für die Potenz eines Medikamentes an. Die nach Hill bezeichnete Gleichung wird für die mathe- matische Darstellung verwendet (HoLFord and SHeINER 1981):

$E=\frac{E_{\max } \cdot C^{n}}{E_{50}{ }^{n}+C^{n}}$

$\mathrm{E}=$ beobachtete Wirkung bei der Konzentration $\mathrm{C} ; \mathrm{n}=$ Exponent, der ein Maß für die Breite des linearen Bereiches in der sigmoidalen Kurve gibt.
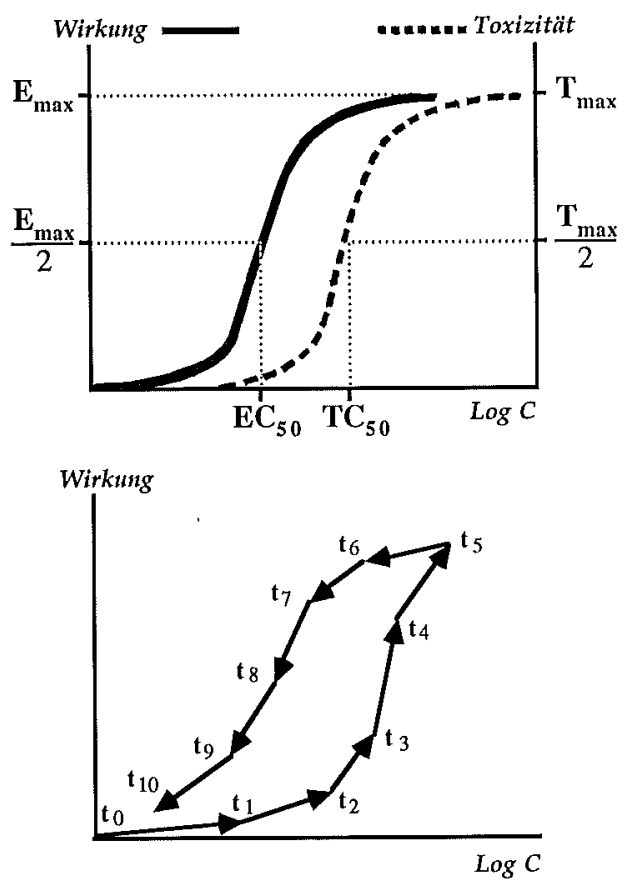

Abb. 11.3. Oben: Klassische Dosis-Wirkungskurve mit den charakteristischen Parametern. Unten: Beispiel zur Dosis-Wirkungskurve mit Hysteresis im Gegenuhrzeigersinn 
Die Maßeinheiten für $\mathrm{E}$ und $\mathrm{E}_{\max }$ sind die, die für das Maß der Wirkung verwendet werden (Unterschied zu einem Basalwert, Prozentsatz des Erfolges, etc). Die Parameter $\mathrm{E}_{\max }, \mathrm{EC}_{50}$ und $\mathrm{n}$ werden durch multiple, nicht lineare Regression ermittelt, wobei die Varianzen mitberücksichtigt werden müssen, da sie ein Maß für die Variabilität der klinischen Wirkung sind.

Das gleiche Modell gilt auch für die Beziehung Plasmakonzentrationen - toxische Wirkung, definiert als "toxische Konzentration 50" ( $\left.\mathrm{TC}_{50}\right)$. Der therapeutische Bereich leitet sich ab von der Differenz $\mathrm{TC}_{50}-$ $\mathrm{EC}_{50}$, - und der therapeutische Index aus dem Verhältnis $\mathrm{EC}_{50} / \mathrm{TC}_{50}$.

\subsubsection{Pharmakokinetiscbes - pharmakodynamisches Modell}

Die Hill'sche Gleichung ist allgemein für steady-state Verhältnisse gültig. Bei einer isolierten Gabe eines Medikamentes kann es aber geschehen, daß die beobachteten Wirkungen unterschiedlich ausfallen, je nachdem ob es sich um die Absorptions- oder
Eliminationsphase (ansteigende oder absinkende Konzentrationen) handelt (CAMPBELL 1990, Oosterhuis und van Boxtel 1988). Die Abb. 11.3 (unten) stellt eine (log)Konzentrations-Response Kurve oder HysteresisKurve dar. Eine Drehung gegen den Uhrzeigersinn kann interpretiert werden als eine Verzögerung zwischen der Diffusion des Medikamentes im Plasma und der auf dem Niveau der Rezeptoren, oder als Einwirkung eines Metaboliten, als Trägheitseffekt oder als Mechanismus der Sensibilisierung. Die Hysteresis im Uhrzeigersinn kann die Folge einer raschen Toleranzentwicklung auf das Medikament oder das Auftreten von Mechanismen der Gegenregulation bedeuten. In diesen Situationen wird ein pharmakokinetisches - pharmakodynamisches Modell verwendet, in dem die Gleichung von Hill nicht auf die Plasmakonzentrationen angewendet wird, sondern auf die Konzentrationen in einem fiktiven Wirkungskompartiment (Abb. 11.1), mit einem scheinbaren Verteilungsvolumen $=\mathrm{O}$, bei dem die durch das Experiment erhaltenen Konstanten $\mathrm{K}_{1 \mathrm{e}}$ und $\mathrm{K}_{\mathrm{e} 0}$ die Hysteresis erklären (OOSTERHuIs und VAN BOXTEL 1988).

\subsection{Konzepte des Drug monitoring}

In der Klinik wird versucht, die Dosierung eines Medikamentes mit dem Ziel eine optimale Wirkung zu erzielen, auf den einzelnen Patienten anzupassen. Manchmal genügt eine grobe Anpassung, wie die Berüicksichtigung des Körpergewichtes. In anderen Fällen ist eine Anpassung an physiologische, pathologische und pharmakologische Gegebenheiten notwendig: Alter des Patienten, Vorliegen einer genetischen Defizienz des Metabolismus, Komedikationen, begleitende Krankheiten. Dies ist vor allem der Fall bei Medikamenten mit geringer therapeutischer Breite, mit steiler Dosis-Wirkungskux- ve, mit großen interindividuellen Wirkungsunterschieden oder auch bei Compliance Problemen. Es ist selten möglich, wie bei den Antikoagulantien, sich direkt auf einen Marker biologischer Aktivität zu beziehen. Die Annahme, daß die interindividuelle Variabilität in der Response auf pharmakokinetische Unterschiede zurückzuführen ist, bildet die Grundlage des drug monitoring, bei dem versucht wird, die Plasmaspiegel innerhalb eines "therapeutischen Bereiches" zu bringen. Dazu mißt man die Plasmaspiegel im steady-state, d. h. im zeitlichen Abstand von etwa vier bis fünf Halbwertszeiten 
seit der letzten Dosisänderung, durch eine Blutabnahme kurz vor der nächsten Dosisverabreichung ("troughlevel"). Verschiedene Strategien bieten sich für die Adaptierung des therapeutischen Schemas mittels Blutspiegelmessungen an (RICHENS und MARKS 1981).

\subsubsection{Dreierregel}

Die Initialdosis ist dem Körpergewicht des Patienten angepaßt. Die anschließend in steady-state Bedingungen erhaltenen Plasmaspiegel werden in Relation zur Zielkonzentration gesetzt, die als Ausgangsbasis für die weitere Dosisanpassung gilt. Diese Strategie ist die im Bereiche der Antidepressiva (Preskorn 1989) und Neuroleptika (BalantGORgIA und BaLANT 1987, DaHL 1986) am häufigsten verwendete, wo hingegen sich das Monitoring von Benzodiazepinen in steady-state Bedingungen noch nicht gerechtfertigt hat (Crismon 1983).

\subsubsection{Pharmakokinetische Studie}

Es werden mehrere Plasmaspiegel zu verschiedenen Zeiten nach der letzten Dosisverabreichung bestimmt, um aus den Ergebnissen graphisch die dem Patienten eigenen pharmakokinetischen Parameter zu ermitteln. Es kann daraus die notwendige Dosierung berechnet werden, um die angestrebte "therapeutische" Gleichgewichtskonzentration $\mathrm{C}_{s s} \mathrm{zu}$ erhalten. Diese Methode wird in der Praxis selten verwendet. Von mehreren Autoren wurde jedoch vorgeschlagen, den 12-, 24- oder 36-Stunden Plasmakonzentrationswert nach einer Einzeldosis des Medikamentes zu verwenden, um daraus die optimale Dosis für eine Dauerbehandlung zur Erreichung einer "therapeutischen" steady-state Konzentration zu erstellen. Dazu sind experimentell erarbeitete Prädiktions- tabellen hilfreich, da eine gute Korrelation zwischen dem erst und letztgenannten Wert gemessen wurde. Solche Verfahren sind für Lithium (AMDISEN und NIELSEN-Kudsk 1986, Rosenberg et al. 1987), Desipramin (BertSCHY et al. 1989, NeLSON et al. 1987), Amitriptylin (Madakasira et al. 1984, DawLing et al. 1980), Maprotilin (Dawling et al. 1980) und Nortriptylin (Geller et al. 1985 , Browne et al. 1983) entwickelt worden, doch werden sie wahrscheinlich selten verwendet. Es muß auch darauf hingewiesen werden, daß gewisse Antidepressiva wie Desipramin oder Imipramin (SINDruP et al. 1990) nicht immer eine lineare Kinetik aufweisen.

\subsubsection{Populationskinetik}

Umfassende klinische Studien haben es für zahlreiche Medikamente ermöglicht, die Bezichung zwischen den persönlichen pharmakokinetischen Parametern eines Patienten mit seinem physiologischen Zustand (Alter, Geschlecht, Gewicht, Leber- und Nierenstatus, medikamentöse Zwischenwirkungen) herzustellen. Für diese Arzneimittel kann die Berücksichtigung dieser Daten zur Initialdosierung hilfreich sein.

\subsubsection{Drug monitoring und Bayes'sche Theorie}

Die kinetischen Populationsstudien liefern auch Informationen zur interindividuellen Variabilität jedes kinetischen Parameters für die untersuchten Medikamente. Wenn ein Plasmaspiegel sich außerhalb des therapeutischen Bereiches befindet, erlaubt die Inferenz-Theorie nach Bayes mit großer Wahrscheinlichkeit individuelle Abweichungen des einen oder andern Parameters dafür verantwortlich zu machen. Jeder Plasmaspiegel modifiziert demnach die kinetischen Parameter der Population, um sie möglichst 
nahe an die des Patienten zu bringen. Aus diesen individualistischen Parametern läßt sich ein auf den einzelnen Patienten abgestimmtes Dosierungsschema ableiten (ScHUMACHER und Barr 1984). In der Psychopharmakologie wurde bisher lediglich für Lithium diese Strategie angewandt (Wiluams et al. 1989). Für gewisse Medikamente gibt es bereits Computerprogramme, die alle drei Strategien berücksichtigen.

\subsubsection{Freie Plasmaspiegel}

Für gewisse Medikamente und unter gewissen Bedingungen je nach Krankheit oder Komedikation, kann die Bestimmung der freien Fraktion des Medikamentes im Plasma indiziert sein, besonders wenn sie sich schlecht in der Gesamtkonzentration widerspiegelt (Levy und Moreland 1984, Tillement et al. 1984). Die analytischen Schwierigkeiten begrenzen jedoch ihren Anwendungsbereich (siehe 11.2.2).

\subsubsection{Speichelbestimmungen}

Speichelbestimmungen hätten in der Klinik wegen ihres nicht invasiven Charakters ihren Platz, wenn sie ein direktes Maß für die freie oder gesamte Plasmakonzentration des Medikamentes $\left(C_{p}\right)$ wären. Tatsächlich gelangen lipophile, nicht ionisierte und nicht an Plasmaeiweiße gebundene Pharmaka allgemein durch Diffusion in den Speichel (Lithium stellt einen Sonderfall bezüglich der Transportmechanismen in den Speichel dar). Doch haben die meisten, für das Monitoring interessanten psychotropen Pharmaka basischen Charakter und liegen im Plasma als Ionen vor. Die Speichelkonzentration $\left(C_{s}\right)$ hängt nach folgender Gleichung von der Dissoziationskonstanten $\mathrm{pK}_{\mathrm{a}}$ der Droge, vom $\mathrm{pH}$ des Plasmas $\left(\mathrm{pH}_{\mathrm{p}}\right)$ und des Speichels $\left(\mathrm{pH}_{\mathrm{s}}\right)$ ab (Muскıо et al. 1978):

$\frac{\mathrm{C}_{\mathrm{s}}}{\mathrm{C}_{\mathrm{p}}}=\frac{1+10^{\left(\mathrm{pK}_{\mathrm{a}-\mathrm{pH}}\right)}}{1+10^{\left(\mathrm{pK}_{\mathrm{a}}-\mathrm{pH} \mathrm{s}\right)}} \times \frac{f_{\mathrm{p}}}{f_{\mathrm{s}}}$

$f_{s}, f_{p}=$ freie Fraktion des Medikamentes im Speichel resp. Plasma.

Da die meisten Medikamente im Speichel nicht gebunden sind $\left(f_{s}=1\right)$, besteht für solche mit einem $\mathrm{pK}_{\mathrm{a}}$-Wert um 9-10 die Beziehung (BaUmann et al. 1982):

$\log \mathrm{C}_{\mathrm{s}}=-\mathrm{pH}_{\mathrm{s}}+7.4+\log$ free $\mathrm{C}_{\mathrm{p}}$

Die freie Plasmakonzentration "free Cp" kann aus den Meßwerten des Speichel-pHWertes und $\mathrm{C}_{s}$ berechnet werden. Speichelbestimmungen haben sich für routinemärSige Anwendungen wegen der oben dargestellten komplizierten Zusammenhänge nicht bewährt, obwohl zahlreiche Psychopharmaka in Forschungsarbeiten im Speichel gemessen wurden (PAXTON 1979).

\section{Literatur}

AMDisen A (1983) Serum level monitoring and clinical pharmacokinetics of lithium. In: GIBAIDI M, PrescotT L (eds) Handbook of clinical pharmacokinetics. Section IV. Therapeutic drug monitoring and dosage prediction. ADIS Health Science Press, New York Tokyo Mexico Sydney Auckland Hong Kong, pp 109-131 AMdisen A, NiEISEN-KUdSk (1986) Relationship bet- ween standardized twelve-hour serum lithium, mean serum lithium of the 24-hour day, dose regimen, and therapeutic interval. An evaluation based on pharmacokinetic simulations. Pharmacopsychiatry 19: 416-419

American College of Clinical Pharmacology: Allen L, Kimura K, MacKichan J, Ritschel WA, AD hoc COMMITTEE FOR PHARMACOKINETIC NOMENCLATURE 
(1982) In: Manual of symbols, equations \& definitions in pharmacokinetics. Philadelphia. Am Coll Clin Pharmacol, pp 1-19

Balant-Gorgia AE, Balant L (1987) Antipsychotic drugs. Clinical pharmacokinetics of potential candidates for plasma concentration monitoring. Clin Pharmacokinet 13: 65-90

Baumann P, Tinguely D, Koeb L, Schöpp J, Le PK (1982) On the relationship between free plasma and saliva amitriptyline and nortriptyline. Int Pharmacopsychiatry 17: 136-146

Baumann P, Eap CB, Müller WE, Tildement JP (eds) (1989) Alpha1-acid glycoprotein. Genetics, biochemistry, physiological functions, and pharmacology. Alan R Liss, New York, p 470

Bertschy G, Vandel S, Vandel B, Aluers G, Bechtel P, Volmht R (1989) Desipramine dose prediction based on 24-hour single-dose levels: feasibility and validity. Pharmacopsychiatry 22 : 161-164

Breyer-Pfaff U, GatrTner HJ (1987) Antidepressiva. Pharmakologie, therapeutischer Einsatz und Klinik der Depression. Medizinisch-pharmakologisches Kompendium, Bd 5. Wissenschaftl Verlagsgesellschaft $\mathrm{mbH}$, Stuttgart, S 252

Browne JL, Perry PJ, Alexander B, Sherman AD, Tsuang MT, Dunner F, PFohl B (1983) Pharmacokinetic protocol for predicting plasma nortriptyline levels. J Clin Psychopharmacol 3: 351-356

Burchel. B, Coughtrie MWH (1989) UDPGlucuronosyltransferases. Pharmacol Ther 43 : 261-289

Campbell DB (1990) The use of kinetic-dynamic interactions in the evaluation of drugs. Psychopharmacology 100: 433-450

CRISMON ML (1983) Benzodiazepine anxiolytics. In: Mungall DR (ed) Applied clinical pharmacokinetics, Raven Press, New York, pp 285-302

DaHL SG (1986) Plasma level monitoring of antipsychotic drugs. Clinical utility. Clin Pharmacolkinet $11: 36-61$

Dawing S, Bralthwiterte R, McAulley R, Montgomery $S$ (1980) Single oral dose pharmacokinetics of amitriptyline and maprotiline in depressed patients. Prediction of steady-state plasma concentrations. In: Beaumont G, Purcell H, Willams NJ (eds) British Journal of Clinical Practice 1980 Symposium (Ciba) Jersey, 79; Supplement 7. New Perspectives in Depressive IlIness. Cradley Heath, Cradley Printing

DobBs JH (1983) The tricyclic antidepressants. In: Mungall DR (ed) Applied clinical pharmacokinetics. Raven Press, New York, pp 271-284
Geller B, Cooper TB, Chestnut EC, Anker JA, Price DT, YATES E (1985) Child and adolescent nortriptyline single dose kinetics predict steady state plasma levels and suggested dose: preliminary data. J Clin Psychopharmacol 5: 154158

Gilman AG, Rall TW, Nies AS, Taylor P (1990) Goodman and Gilman's: the pharmacological basis of therapeutics. Pergamon Press, New York

Grennblatt DJ, Divoll M, Abernethy DR, Ochs HR, Shader RI (1983) Clinical pharmacokinetics of the newer benzodiazepines. Clin Pharmacokinet 8: 233-252

GUENTFRT TW (1984) Pharmacokinetics of benzodiazepines and of their metabolites. Prog Drug Metabol 8: 241-386.

HOLFORD NHG, SHeINER IAB (1981) Understanding the dose-effect relationship: clinical application of pharmacokinetic-pharmacodynamic models. Clin Pharmacokinet 6: 429-453

Jørgensen A (1986) Metabolism and pharmacokinetics of antipsychotic drugs. Prog Drug Metabol 9: 111-174

Kiotz U (1983) Individualisierung der Medikamentendosierung durch Arzneimittelmessungen im Plasma und Anwendung pharmakokinetischer Prinzipien. J Clin Chem Clin Biochem 21: 649-658

LEVY RH, MORELAND TA (1984) Rationale for monitoring free drug levels. Clin Pharmacokinet 9 [Suppl 1]: 1-9

Madakasira S, Khazanie PG, Sato TL (1984) Single dose single-point method in amitriptyline therapy. Psychopharmacology 84: 574-576

MuckLOW JC, BENDING MR, KAHN GC, DOLLeRY CT (1978) Drug concentration in saliva. Clin Pharmacol Ther 24: 563-570

Nelson JC, Jatlow PI, Mazure C (1987) Rapid desipramine dose adjustment using 24-hour levels. J Clin Psychopharmacol 7: 72-77

Oosterhuis B, van Boxtel CJ (1988) Kinetics of drug effects in man. Ther Drug Monit 10: 121-132

PAXION JW (1979) Measurement of drugs in saliva: a review. Methods Find Exp Clin Pharmacol 1: 11-21

PIAFSKY KM (1983) Disease-induced changes in the plasma binding of basic drugs. In: GIBALDI $M$, Prescott L (eds) Handbook of clinical pharmacokinetics. Section III. Effect of disease states on drug disposition. ADIS Health Science Press, New York Tokyo Mexico Sydney Auckland Hong Kong, pp 70-88

PresKonN SH (1989) Tricyclic antidepressants: the whys and hows of therapeutic drug monitoring. J Clin Psychiatry 50 [Suppl 7]: $34-42$ 
ReyntJens AJM, Heykants JJP, Woestenborghs RJH, GeLDERS YG, ABRTS TJL (1982) Pharmacokinetics of haloperidol decanoate. A two-year follow-up. Int Pharmacopsychiatr 17: 238-246

Richins A, Marks V (eds) (1981) Therapeutic drug monitoring, 1st ed. Churchill Livingstone, London

Rosenberg JG, Binder RL, Berlant J (1987) Prediction of therapeutic lithium dose: comparison and improvement of current methods. J Clin Psychiatry 48: 284-286

Rowland M, Tozer TN (eds) (1989) Clinical pharmacokinetics: concepts and applications. Lea \& Febiger, Philadelphia, p 331

SCHUMACHER GE, BARr JT (1984) Bayesian approaches in pharmacokinetic decision making. Clin Pharm 3: 525-530

Sindrup SH, Brosen K, Gram LF (1990) Nonlinear kinetics of imipramine in low and medium plasma level ranges. Ther Drug Monit 12: 445449
Tillement JP, Houin G, Zin R, Urien S, Albengres E, Barre J, Lecomte M, D'Athis P, Sebille B (1984) The binding of drugs to blood plasma mactomolecules: recent advances and therapeutic significance. Adv Drug Res 13: 59-94

Tillement' JP, Lindenlaub E (eds) (1985) Protein binding and drug transport. Symposium Alvor, Algarve, Portugal, Sept. 85. Symposia Medica Hoechst 20. Schattauer, Stuttgart New York, p 530

Tozer TN (1981) Concepts basic to pharmacokinetics. Pharmacol Ther 12: 109-131

Wandeli. M, Wilcox-Thole WL (1983) Protein binding and free drug concentration. In: MungaLL. DR (ed) Applied clinical pharmacokinetics. Raven Press, New York, pp 17-48

Williams PJ, Browne JL, PaTel RA (1989) Bayesian forecasting of serum lithium concentrations. Comparison with traditional methods. Clin Pharmacokinet 17: 45-52 